\title{
REGRESSION MODELS WITH MEMORY FOR THE LINEAR RESPONSE OF TURBULENT DYNAMICAL SYSTEMS*
}

\author{
EMILY L. KANG ${ }^{\dagger}$, JOHN HARLIM ${ }^{\ddagger}$, AND ANDREW J. MAJDA ${ }^{\S}$
}

\begin{abstract}
Calculating the statistical linear response of turbulent dynamical systems to the change in external forcing is a problem of wide contemporary interest. Here the authors apply linear regression models with memory, $\mathrm{AR}(\mathrm{p})$ models, to approximate this statistical linear response by directly fitting the autocorrelations of the underlying turbulent dynamical system without further computational experiments. For highly nontrivial energy conserving turbulent dynamical systems like the Kruskal-Zabusky (KZ) or Truncated Burgers-Hopf (TBH) models, these AR(p) models exactly recover the mean linear statistical response to the change in external forcing at all response times with negligible errors. For a forced turbulent dynamical system like the Lorenz-96 (L-96) model, these approximations have improved skill comparable to the mean response with the quasi-Gaussian approximation for weakly chaotic turbulent dynamical systems. These $\operatorname{AR}(\mathrm{p})$ models also give new insight into the memory depth of the mean linear response operator for turbulent dynamical systems.
\end{abstract}

Key words. Fluctuation-dissipation theory, autoregressive models, linear response, climate change.

AMS subject classifications. 37N10, 76F 20 .

\section{Introduction}

One of the cornerstones of modern statistical physics is the fluctuation-dissipation theorem (FDT), which roughly states that for systems of identical particles in statistical equilibrium, the average response to small external perturbations can be calculated through the knowledge of suitable correlation functions of the unperturbed statistical system. For some of the many practical applications of the FDT, see $[24,6]$. The low frequency response to changes in external forcing for various components of the climate is a central problem of contemporary climate change science. Leith [25] suggested that if the climate system satisfied a suitable FDT then the climate response to small external forcing should be calculated by estimating suitable statistics in the present climate. The climate system is a forced dissipative chaotic dynamical system which is physically quite far from the classical physicist' setting for FDT. Leith's suggestion has created a lot of recent activity in generating new theoretical formulation $[28,40,32,13,27,18]$ and approximate algorithms for FDT with applications to climate response $[16,17,14,15,1,2,3,4]$. FDT combined with empirical information theory leads to new strategies for correcting imperfect models in a training phase where observations are available to have improved long range low frequency forecasting skill $[29,30,31]$.

The simplest approximate strategies for computing the response of chaotic turbulent dynamical systems use a linear regression model $[41,10]$ to fit the statistics of Cai.

${ }^{*}$ Received: November 29, 2011; accepted (in revised form): July 1, 2012. Communicated by David

${ }^{\dagger}$ Department of Mathematical Sciences, University of Cincinnati, Cincinnati, OH 45221, USA (kangel@ucmail.uc.edu).

${ }^{\ddagger}$ Department of Mathematics, North Carolina State University, Box 8205, Raleigh, NC 27695, USA (jharlim@ncsu.edu).

$\S$ Department of Mathematics and Center for Atmospheric and Ocean Science, Courant Institute of Mathematical Sciences, New York University, New York, NY 10012, USA (jonjon@cims.nyu.edu). 
the nonlinear dynamics,

$$
\frac{d \mathbf{u}_{R}}{d t}=L_{R} \mathbf{u}_{R}+\sigma_{R} \dot{W}+F,
$$

where $\dot{W}$ is a vector white noise and $L_{R}$ has eigenvalues with negative real part. Since $L_{R}$ has decaying spectrum, the linear operator in (1.1) has its own linear regression FDT [12] and one strategy [42] is to use the prediction of FDT for this approximate model as a surrogate for the actual linear response operator, which has a vastly larger computational overhead [28]. However, there are inherent realizability issues so that there often is not a linear operator, $L_{R}$, with negative real eigenvalues which reproduces the lagged covariances [41, 9] so that there are severe model errors [32] in calculating the linear response. A different linear regression strategy involving utilizing Ornstein-Uhlenbeck processes introduced recently [32], the Mean Stochastic Model (MSM), is guaranteed to always be realizable by matching the variances and the integral of the lagged covariances with data from the turbulent dynamical system (see Section 2 below); the matching of the integral of the covariance guarantees the best infinite time response of the linear regression model in reproducing the actual FDT response to the change in forcing. There are extensive comparisons [32] between MSM and the earlier standard single step regression strategy [41, 10] demonstrating the clear superiority of MSM for estimating the mean statistical response operator for changes in mean forcing.

The goal of the present paper is to build linear regression models with memory [ 5 , $7,22]$, called AR(p) models, to improve the skill of the finite time mean linear response to the change in external forcing through such models. Besides the improved response at finite times over MSM, this analysis leads to an assessment of the memory effects in the system without performing detailed simulations of the turbulent dynamical system to test this. Nevertheless, as pointed out in $[32,30]$ all linear regression models including MSM and $\mathrm{AR}(\mathrm{p})$ always generate Gaussian statistics and necessarily always yield a zero variance response to the change in external forcing; even when the actual variance response in the turbulent dynamical system is large $[28,1,3]$. Thus, there is an intrinsic information barrier in all linear regression model approximations for the linear response of the variance of turbulent dynamical systems [29, 30, 31].

With this background, the outline of the present paper is the following. Section 2 contains a brief summary of FDT and the MSM and AR(p) linear regression algorithms. Section 3 contains the application of MSM and AR(p) linear regression algorithms to the finite time linear response of three energy conserving turbulent dynamical systems: the Kruskal-Zabusky (KZ), truncated Burgers-Hopf (TBH), and inviscid-unforced Lorenz-96 (IL96) models [28]; the improved skill of AR(p) over MSM in the finite time statistical mean response to external forcing is especially significant for the KZ model with highly oscillatory multi-scale correlation functions. For these energy conserving turbulent dynamical systems, $\mathrm{AR}(\mathrm{p})$ essentially recovers the mean linear response to external forcing at all times exactly with negligible errors. In Section 4, the $\mathrm{AR}(\mathrm{p})$ regression models are applied to the forced dissipative L-96 model $[26,28]$. Here the skill of the AR(p) models for linear response of weakly chaotic turbulent dynamical systems (with forcing strengths $F=5,6$ ) at moderate times is superior to MSM and comparable to the quasi-Gaussian FDT approximation at vastly cheaper computational cost. Section 5 is a brief concluding discussion. 


\section{Fluctuation-dissipation theory and linear regression models}

In this section, we provide a brief informal summary of FDT; see [28] for more details. Our discussion emphasizes how to compute the response operator to a constant change in forcing with Gaussian invariant probability measure. We also discuss two linear regression models (one with memory) for speeding up the computation of the linear response operator.

2.1. Fluctuation-dissipation theory. Consider a chaotic nonlinear dynamical system of ordinary differential equations for a state vector $\mathbf{u} \in \mathbb{R}^{N}$, given by

$$
\frac{d \mathbf{u}}{d t}=\mathbf{f}(\mathbf{u})
$$

Let $A(\mathbf{u})$ be a linear or nonlinear functional of interests; in the present paper $A(\mathbf{u})=\mathbf{u}$ is the mean state response. The average value $\langle A\rangle$ of $A(\mathbf{u})$ for the dynamics in (2.1) is computed as a time average along a single long-term trajectory of (2.1):

$$
\langle A\rangle=\lim _{r \rightarrow \infty} \frac{1}{r} \int_{0}^{r} A[\mathbf{u}(t)] d t .
$$

We assume that the dynamical system in (2.1) is perturbed by small external forcing as

$$
\frac{d \mathbf{v}}{d t}=\mathbf{f}(\mathbf{v})+\delta \mathbf{f}(t) .
$$

In our framework, the external forcing represents the collection of altered parameters (such as solar radiative forcing) that cause external change. Apparently, the solution $\mathbf{v}(t)$ will cause $\langle A\rangle$ to deviate from its original value, which represents the average response to the change in the external parameters:

$$
\delta\langle A\rangle=\lim _{r \rightarrow \infty} \frac{1}{r} \int_{0}^{r}\{A[\mathbf{v}(t)]-A[\mathbf{u}(t)]\} d t .
$$

The formula in (2.3) provides a straightforward estimate for the average response, based on the distance between two different numerically simulated long-term trajectories of the perturbed and unperturbed models, respectively. However, it requires separate long-term numerical simulations of the turbulent dynamical system for each perturbation, which can be numerically expensive. Also, it computes the average response at infinite time, and provides no information about transient time-dependent behavior of average response when the changes in external forcing are applied to the dynamics.

Provided that the change in external forcing is small, the fluctuation-dissipation theorem [28] offers a more versatile way to construct an approximation to $\delta\langle A\rangle$ :

$$
\delta\langle A\rangle(t)=\int_{0}^{t} R(s) \delta \mathbf{f}(t-s) d s,
$$

where $t=0$ is the initial time when the forcing change is introduced into the dynamical system. Here, $R(t)$ is the response operator, which directly relates the changes in external parameters $\delta \mathbf{f}$ to the average response $\delta\langle A\rangle$. Note that $R(t)$ does not depend on the magnitude and direction of $\delta \mathbf{f}$ and, once computed, provides the response for a wide range of magnitudes and directions $\delta \mathbf{f}$. Also, one can study the singular 
directions of $R$ to determine which changes in $\delta \mathbf{f}$ will result in a most catastrophic response of $\delta\langle A\rangle[28,40]$. Additionally, the formula in (2.4) provides the time-dependent climate response for finite values of $t$, which allows the study of transient response effects. In the situation when the forcing $\delta \mathbf{f}$ is a constant (which is "turned on" at time $t=0)$ and does not depend on space and time, a simplified version of (2.4) is used:

$$
\delta\langle A\rangle(t)=\mathcal{R}(t) \delta \mathbf{f}, \quad \mathcal{R}(t)=\int_{0}^{t} R(s) d s .
$$

In the numerical experiments throughout the paper, a constant change in forcing $\delta \mathbf{f}$ is used. Evaluating the response operator $R(t)$, or its constant change in forcing counterpart $\mathcal{R}(t)$, is a nontrivial problem.

If the invariant measure of the unperturbed dynamical system in (2.1) is Gaussian with mean state $\overline{\mathbf{u}}$ and covariance matrix $C$, the response operator $R(t)$ is evaluated as a correlation function $[8,43,28]$ :

$$
R_{G}(t)=\lim _{r \rightarrow \infty} \int_{0}^{r} A[\mathbf{u}(t+s)] C^{-1}(\mathbf{u}(s)-\overline{\mathbf{u}}) d s
$$

Notice that although the formula in (2.6) is defined for Gaussian invariant measures, the actual average is computed using solutions of the nonlinear dynamical system in (2.1) assuming that the dynamics are strongly mixing and dissipative. When the unperturbed dynamical system in (2.1) has an invariant measure that is not Gaussian, then the response operator in (2.6) is understood as the "quasi-Gaussian approximation for FDT" [25] and we call it the qG-FDT response operator in this article.

As we discussed in the introduction, if we apply (2.6) to compute the variance response operator to the change in external forcing with quadratic functional $A(\mathbf{u})=$ $(\mathbf{u}-\overline{\mathbf{u}})^{T} Q(\mathbf{u}-\overline{\mathbf{u}})$ for any constant $Q$, we obtain zero variance response since the Gaussian statistics have zero third moment even if the true variance response may be large $[28,1,3]$. As we mentioned earlier, we only consider the mean response to the change in external forcing with functional $A(\mathbf{u})=\mathbf{u}$; in this case, evaluation of $R_{G}(t)$ in (2.6) is simply an autocorrelation function.

The goal of this paper is to show that we can approximate the mean response operator in (2.6) with significantly reduced computational cost by averaging it with respect to solutions of much simpler linear stochastic models relative to solutions of (2.1) provided that the two systems are statistically consistent. In the remaining parts of this section, we discuss two linear stochastic regression models with Gaussian equilibrium measures (one of them with memory) that are fitted to the equilibrium statistics of the nonlinear dynamics in (2.1). Then, we use solutions of these regression models to compute the mean response operator $R_{G}(t)$.

2.2. Mean stochastic models. The Mean Stochastic Model (MSM) [32] is an alternative regression fitting to the linear stochastic model in (1.1). Consider a projection of $\mathbf{u}$ on a spectral coordinate $\mathbf{u}$ through a linear transformation $\mathcal{F}$. For example, the linear operator $\mathcal{F}$ could involve the empirical orthogonal functions (EOF) basis or even the spherical harmonics basis if components of $\mathbf{u}$ represent variables that are appropriately distributed in spherical geometry. In our application below, we consider components of $\mathbf{u} \in \mathbb{R}^{N}$ as scalar quantities at equally distributed points on a unit circle; in this simple setup, the operator $\mathcal{F}$ is simply a discrete Fourier transform. 
The MSM involves statistical solutions of a constantly forced Ornstein-Uhlenbeck process on each Fourier coefficient:

$$
d \hat{u}_{k}=\left(-\gamma_{k}+\mathrm{i} \omega_{k}\right) \hat{u}_{k} d t+f_{k} d t+\sigma_{k} d W_{k}(t), \quad|k| \leq N / 2 .
$$

In (2.7), complex valued noise is defined as $W_{k}(t)=\left(W_{1}(t)+\mathrm{i} W_{2}(t)\right) / \sqrt{2}$, where each component $W_{i}(t)$ is a standard Wiener process with variance $t / 2$. There are four parameters to be specified for each wavenumber $k$ : the damping coefficient $\gamma_{k}>0$, the frequency $\omega_{k}$, the constant forcing strength $f_{k}$, and the noise strength $\sigma_{k}$.

The differential equation in (2.7) has a Gaussian equilibrium probability measure with mean, covariance (or energy spectrum), and autocorrelation times [12, 34, 32]:

$$
\begin{aligned}
\left\langle\hat{u}_{k}\right\rangle & =\frac{f_{k}}{\gamma_{k}-\mathrm{i} \omega_{k}}, \\
C_{k} & \equiv \lim _{t \rightarrow \infty}\left\langle\left(\hat{u}_{k}(t)-\left\langle\hat{u}_{k}\right\rangle\right)\left(\hat{u}_{k}(t)-\left\langle\hat{u}_{k}\right\rangle\right)^{*}\right\rangle=\frac{\sigma_{k}^{2}}{2 \gamma_{k}}, \\
T_{\text {corr }}(k) & \equiv \lim _{t \rightarrow \infty} \int_{0}^{\infty}\left\langle\left(\hat{u}_{k}(t+\tau)-\left\langle\hat{u}_{k}\right\rangle\right) C_{k}^{-1}\left(\hat{u}_{k}(t)-\left\langle\hat{u}_{k}\right\rangle\right)^{*}\right\rangle d \tau=\frac{1}{\gamma_{k}+\mathrm{i} \omega_{k}} .
\end{aligned}
$$

The MSM regression fitting solves the four equations in (2.8) (notice that $T_{\text {corr }}$ has real and imaginary parts) with empirical statistics $\left\langle\hat{u}_{k}\right\rangle, C_{k}$, and $T_{\text {corr }}$, obtained by averaging (temporally) solutions of the nonlinear dynamics in (2.1). Notice that the MSM regression fitting in (2.8) always guarantees realizable solutions with $\gamma_{k}>$ 0 as pointed out in the introductory section and in [32]; this realizability is not guaranteed for standard linear regression strategies that typically parameterize $\omega_{k}$ with a linearized frequency $[41,10]$.

Since this stochastic model perfectly matches the equilibrium autocorrelation time in (2.8), then by design it should be obvious to the reader that the infinite time mean response operator to a constant change in forcing, $\mathcal{R}(\infty)$, in (2.5) computed with solutions of this linear regression model exactly equals the infinite time qG-FDT response operator. In the present article, we denote the mean response operator computed using the formula in (2.6) with solutions of the MSM as $R_{L R}(t)$, or $\mathcal{R}_{L R}(t)$ for its constant change in forcing counterpart.

2.3. AR(p) models. The autoregressive representation of a stationary time series has been widely used $[7,22]$. In our context, we consider fitting solutions of the nonlinear differential equations in (2.1) to the following constantly forced complex valued autoregressive model of order $p$ (we refer to it as the $\mathrm{AR}(\mathrm{p})$ model in this article) on each Fourier coefficient:

$$
\hat{u}_{k, m}=\phi_{1} \hat{u}_{k, m-1}+\phi_{2} \hat{u}_{k, m-2}+\cdots+\phi_{p} \hat{u}_{k, m-p}+f_{k}+\eta_{k, m}, \quad|k| \leq N / 2 .
$$

In (2.9), index $k$ denotes wavenumber, index $m$ denotes discrete time $t_{m+1}=t_{m}+$ $\Delta t$, and parameter $p \in \mathbb{Z}$ is called the order of this autoregressive model. Larger $p$ corresponds to longer memory depth in the time series that we are fitting. We shall see in the next two sections that $p$ can be significantly different for various wavenumbers depending on the correlation functions and also for various strength of forcing in a forced dissipative chaotic dynamical system.

In (2.9), $\eta_{k, m}$ is a complex valued i.i.d. Gaussian noise with mean zero and variance $C_{k}$. For each wavenumber, we have $p+3$ parameters to be specified, including the constant forcing term that can be fitted directly to the empirical mean state, $\left\langle\hat{u}_{k}\right\rangle$, 
coefficients $\left\{\phi_{j}, j=1, \ldots, p\right\}$, the noise covariance $C_{k}$, and the autoregressive model order $p$. In the present article, we fit parameters $\left\{\phi_{j}, j=1, \ldots, p\right\}$ and $C_{k}$ with the Yule-Walker estimators [7] and choose $p$ based on the FPE criterion [5]. We refer to the Appendix in [22] for detailed discussion of this regression fitting.

Note that the AR(p) model has a Gaussian invariant probability measure and therefore the autocorrelation function of the solutions of this model is essentially the mean response operator as described in (2.6) with functional $A(\mathbf{u})=\mathbf{u}$. We denote the mean response operator computed using the formula in (2.6) with solutions of $\operatorname{AR}(\mathrm{p})$ model as the $R_{A R}(t)$, or $\mathcal{R}_{A R}(t)$ for its constant change in forcing counterpart.

We avoid the MSM and AR(p) models in physical space to minimize the number of parameters to be fitted. Second, in our numerical examples in Sections 3 and 4, the dynamical models are translation invariant so that the linear response operator is block diagonal [28] in Fourier space; this implies cheaper computational cost in Fourier space than in physical space. As we mentioned in the beginning of Section 2.2, one can consider various spectral coordinates depending on the the spatial distribution of the observations.

3. Calculating the linear response of energy conserving turbulent dynamical systems through $\mathrm{AR}(\mathrm{p})$ memory models

In this section, we compute the mean linear response operator to a constant change in external forcing $\delta \mathbf{f}$ on three prototype energy conserving nonlinear chaotic dynamical systems. These three models, the Kruskal-Zabusky, the truncated BurgersHopf, and the inviscid-unforced Lorenz-96, have Gaussian invariant measures with zero mean and equipartition energy [28], but they have very different response characteristics with multiple timescales. In particular, we compare the response operator computed with solutions of the original dynamics $\mathcal{R}(t)$ with those computed with solutions of the MSM and $\operatorname{AR}(\mathrm{p})$ models, $\mathcal{R}_{L R}(t)$ and $\mathcal{R}_{A R}(t)$, respectively.

3.1. Kruskal-Zabusky (KZ) model. The Kruzkal-Zabusky (KZ) model was introduced to numerically study soliton interactions with additional dispersion terms [44]. When these dispersion terms are removed, the governing equation is given by

$$
\frac{d u_{j}}{d t}=-\frac{1}{\Delta x}\left(F_{j+1 / 2}-F_{j-1 / 2}\right), \quad j=1, \ldots, N
$$

where $F_{j+1 / 2}=\left(u_{j}^{2}+u_{j} u_{j+1}+u_{j+1}^{2}\right) / 6$, and periodic boundary conditions $u_{j+N}=u_{j}$. In the present context, we set $N=40$ following the study in $[36,28]$ to ensure the dynamical system is ergodic and strongly mixing with decaying correlations. We numerically integrate the $\mathrm{KZ}$ model with the fourth order Runge-Kutta time integrator with small enough time step $\delta t=5 \times 10^{-4}$ to ensure the energy is conserved [36] and randomly drawn initial condition from the uniform distribution on a constant energy surface $E=1$. We parameterize both the MSM and AR(p) models with solutions of the KZ model in (3.1) for total time $10^{5}$ as the training dataset. Specifically, the parameters in the MSM model in (2.7) are estimated by solving (2.8), with empirical mean and correlation times computed from the training dataset. For the AR(p) model, we estimate the parameters with the same data set with discrete time step $\Delta t=0.02$ (we found worse solutions with smaller $\Delta t$ ). More details on this regression fitting can be found in the Appendix of [22].

First, we report how the MSM and $\mathrm{AR}(\mathrm{p})$ models reproduce the climatological statistics in (2.8), including the energy spectrum, $C_{k}$, the correlation time, $T_{\text {corr }}$, the 

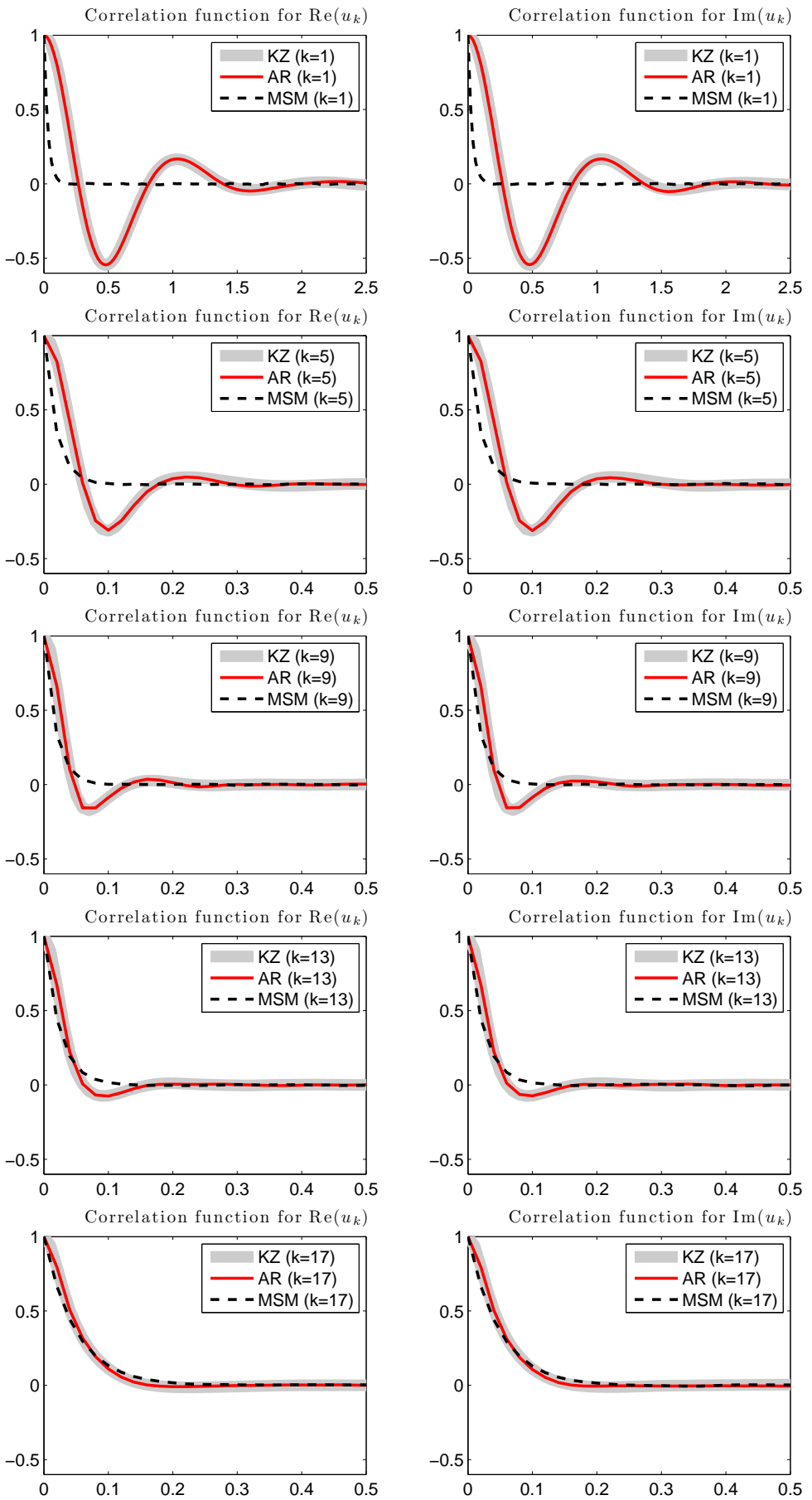

FIG. 3.1. The autocorrelation functions from the $K Z$ model (thick grey solid curves), reproduced from the MSM model (black dashed curves) and the $A R(p)$ model (red solid curves), respectively. 
autocorrelation functions,

$$
\operatorname{Corr}(\tau ; k)=\left\langle\left(\hat{u}_{k}(t+\tau)-\left\langle\hat{u}_{k}\right\rangle\right) C_{k}^{-1}\left(\hat{u}_{k}(t)-\left\langle\hat{u}_{k}\right\rangle\right)^{*}\right\rangle,
$$

and the absolute correlation time

$$
T_{a b s}(k)=\int_{0}^{\infty}|\operatorname{Corr}(\tau ; k)| d \tau,
$$

where the infinite time integrals in (2.8), (3.3) are approximated by long time solutions up to a finite time $T=10^{5}$.

Both the MSM and $\operatorname{AR}(\mathrm{p})$ models reproduce the energy spectrum $C_{k}$ well (the results are not shown). The autocorrelation functions for various wavenumbers in the KZ model are shown in figure 3.1. Notice that the autocorrelation functions evolve slowly and are highly oscillatory for lower wavenumbers. This oscillatory correlation behavior is absent on higher wavenumbers $(k \geq 17)$. As shown in figure 3.1, the $\operatorname{AR}(\mathrm{p})$ model accurately reproduces the autocorrelation function on every wave number. On the other hand, the MSM models cannot reproduce the oscillatory decaying correlation function for low wavenumbers (we show $k=1,5,9$, and 13 in figure 3.1). In figure 3.2, notice that the AR(p) model also reproduces the correlation time, $T_{\text {corr }}$, as well as the absolute correlation time, $T_{a b s}$, with negligible errors. The MSM model, which by design matches the $T_{\text {corr }}$, fails to capture $T_{a b s}$ for low wavenumbers since there is a strong oscillation in the autocorrelation function with negative portion that almost cancels out the positive portions (see figure 3.1).
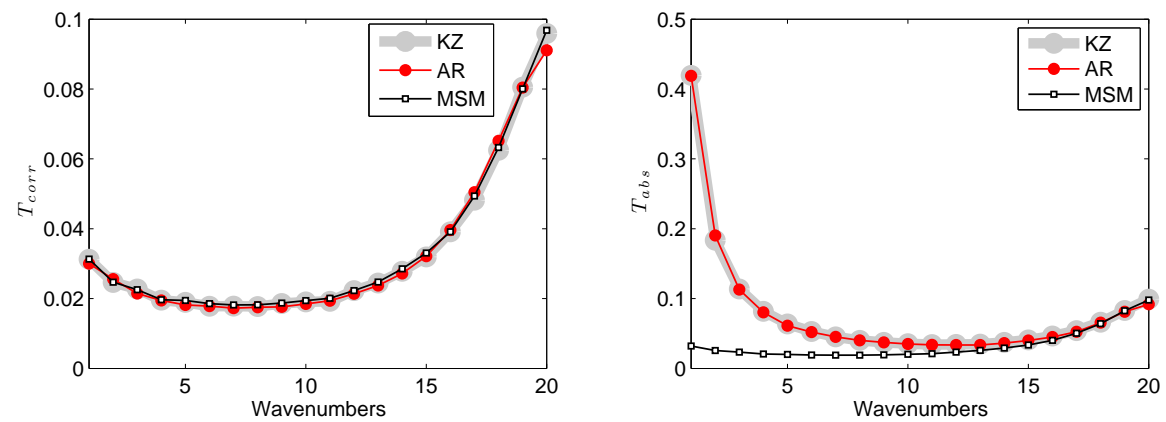

FIG. 3.2. The spectra of correlation times for the $K Z$ model (thick grey solid), reproduced from the MSM model (black dash with squares) and the $A R(p)$ model (red solid with dots). The left panel shows $T_{\text {corr }}$ defined as the integrals with the autocorrelation functions directly. The right panel shows $T_{a b s}$ defined as the integrals with the absolute values of the autocorrelation functions.

In figure 3.3, we show the corresponding order $p$ as a function of wavenumber in the $\mathrm{AR}(\mathrm{p})$ model used to reproduce the climatological statistics in (2.8), (3.2), (3.3). Notice that there is almost (not exactly) one-to-one relation between $p$ and $T_{a b s}$ (compare figure 3.2 with 3.3 ); $T_{a b s}$ and $p$ of wavenumber 1 are roughly three times of those of wavenumbers 2 and 3 , and so on. This observation suggests that the memory depth in wavenumbers with oscillatory and slowly decaying autocorrelation function (such as the lowest wavenumber of the KZ model) is implicitly inferred with a large value of $p$ in the autoregressive modeling. This implicit memory assessment by knowing only the order $p$ in the autoregressive model is advantageous to the standard assessment with $T_{\text {corr }}$ or $T_{a b s}$ in (2.8) or (3.3), which requires more simulations. 


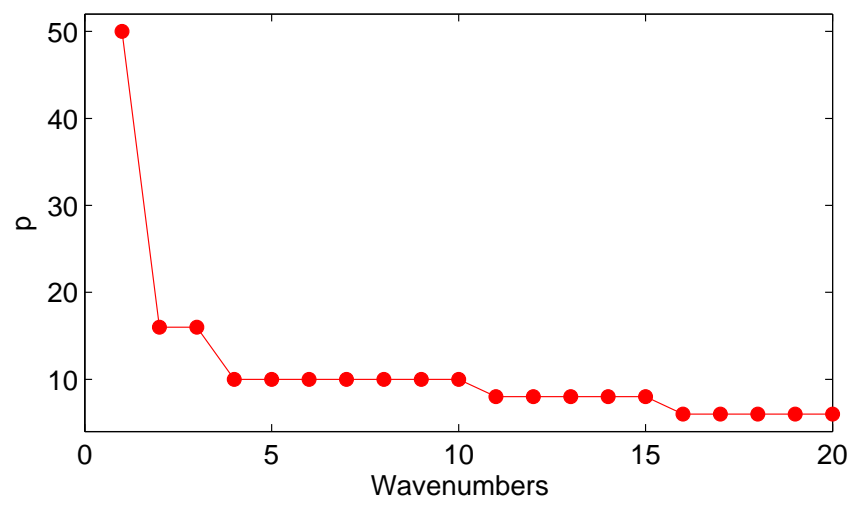

FIG. 3.3. The values of $p$ chosen in the $A R(p)$ model for the $K Z$ model.

Using the solutions from the MSM and AR(p) models, we compute the mean linear response operators to a constant change in forcing, $\mathcal{R}_{L R}(t)$ and $\mathcal{R}_{A R}(t)$, respectively. We compare them with the response operator $\mathcal{R}(t)$ computed with the true solutions of the KZ model. To quantify the performance, we compute the $\ell^{2}$ relative error,

$$
\mathcal{E}_{2}=2 \frac{\left\|\mathcal{R}_{M}(t)-\mathcal{R}(T)\right\|}{\left\|\mathcal{R}_{M}(t)\right\|+\|\mathcal{R}(t)\|},
$$

and the pattern correlation,

$$
C\left(\mathcal{R}_{M}(t), \mathcal{R}(t)\right)=\frac{\left\langle\mathcal{R}_{M}(t), \mathcal{R}(t)\right\rangle}{\left\|\mathcal{R}_{M}(t)\right\| \cdot\|\mathcal{R}(t)\|},
$$

between the true FDT response, $\mathcal{R}(t)$, and the approximate response $\mathcal{R}_{M}(t)$ which can be $\mathcal{R}_{L R}(t)$ and $\mathcal{R}_{A R}(t)$; the norm above, $\|\cdot\|=\langle\cdot, \cdot\rangle^{1 / 2}$, is defined with the standard $\ell^{2}$ inner product $\langle g, h\rangle=\sum_{j=1}^{N} g_{j} h_{j}^{*}$ for $N$-dimensional complex valued vectors $g$ and $h$. Smaller $\ell^{2}$-error and larger pattern correlation indicate better estimate of the linear response operator in terms of amplitude and pattern, respectively.

TABLE 3.1. $\ell^{2}$-errors and correlations between the response operators from the $K Z$ model and those from the $A R(p)$ and the MSM models at different times.

\begin{tabular}{lcccc}
\hline \hline \multirow{2}{*}{ Time } & \multicolumn{2}{c}{$\ell^{2}$-errors } & \multicolumn{2}{c}{ Correlations } \\
& $\mathrm{AR}(\mathrm{p})$ & $\mathrm{MSM}$ & $\mathrm{AR}(\mathrm{p})$ & $\mathrm{MSM}$ \\
\hline 0.12 & 0.0026 & 0.5664 & 1.0000 & 0.8874 \\
0.24 & 0.0085 & 0.6528 & 1.0000 & 0.8324 \\
0.72 & 0.0411 & 0.2299 & 0.9993 & 0.9737 \\
$\infty$ & 0.0408 & 0.0453 & 0.9994 & 0.9995 \\
\hline \hline
\end{tabular}

In figure 3.4, we show the translationally averaged (about the perturbation location) linear response operators in physical space for various times. The linear response in physical space is concentrated at the gridpoint of perturbation with oscillations around it. We find that there is almost no visual difference between response from the $\mathrm{AR}(\mathrm{p})$ model, $\mathcal{R}_{A R}(t)$, and that from the $\mathrm{KZ}$ model, $\mathcal{R}(t)$. Notice in table 3.1 that 

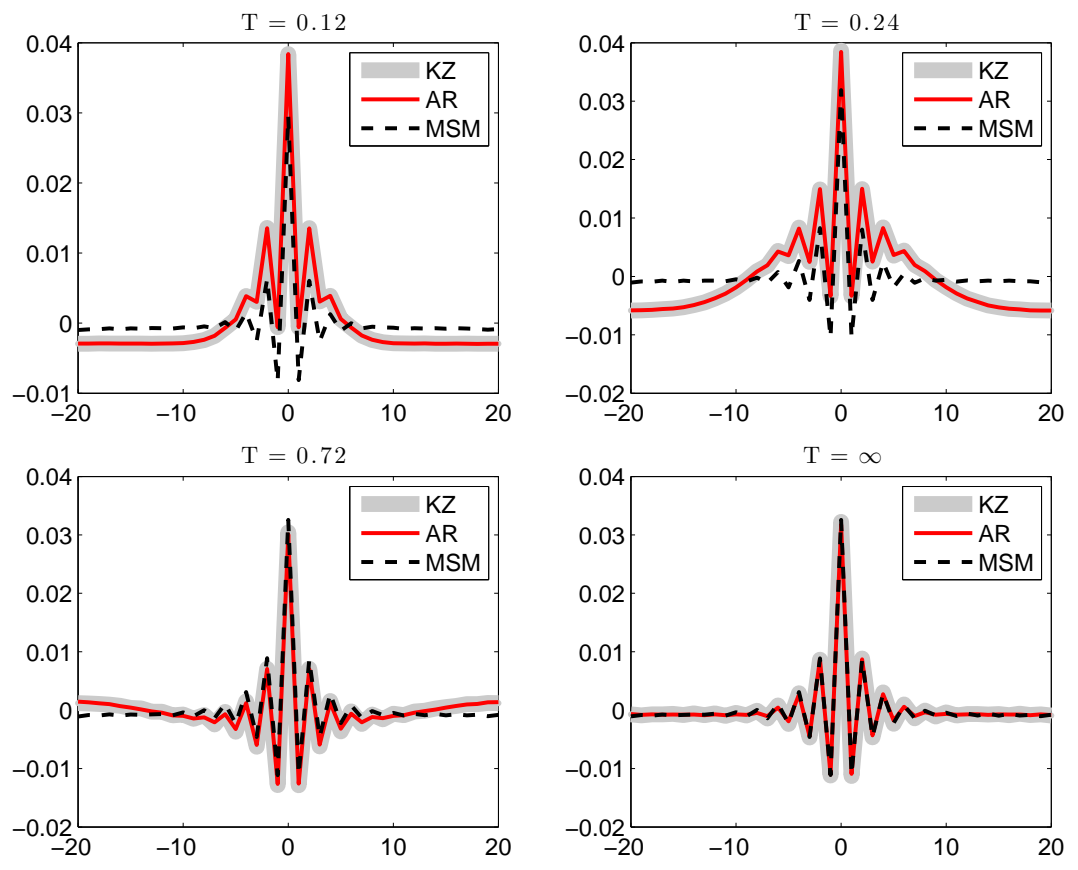

FIG. 3.4. Translationally averaged linear response operators in physical space of the KZ model (thick grey solid lines), the AR(p) model (red solid lines), and the MSM model (black dashed lines) at different times $T=0.12$ (upper-left), 0.24 (upper-right), 0.72 (lower-left), and $\infty$ (lower-right).

the $\ell^{2}$-errors between $\mathcal{R}_{A R}(t)$ and $\mathcal{R}(t)$ are negligible and the corresponding pattern correlations are close to 1 . The response operator $\mathcal{R}_{L R}(t)$, on the other hand, is not accurate at all for shorter times $t=0.12,0.24$ (see the much larger errors in table 3.1 and the response operator mismatches in figure 3.4). The response operator reproduced from the MSM model only performs well at infinite time, which is expected by the regression fitting in (2.8).

3.2. Truncated Burgers-Hopf (TBH) and inviscid-unforced Lorenz-96 (IL96) models. The truncated Burgers-Hopf model (TBH) is a Fourier Galerkin approximation to the inviscid Burgers equation which has intrinsic stochastic dynamics with strong numerical evidence of ergodic and mixing for large enough degrees of freedom (we'll set $N=40$ as in the KZ model and in [28]). The governing equations of its Fourier coefficients are as follows:

$$
\frac{d \hat{u}_{k}}{d t}=-\frac{\mathrm{i} k}{2} \sum_{\substack{k+p+q=0 \\|p|,|q|<N / 2}} \hat{u}_{p}^{*} \hat{u}_{q}^{*}, \quad \hat{u}_{-k}=\hat{u}_{k}^{*}, \quad|k| \leq N / 2 .
$$

Following [36], we numerically integrate the TBH model with a pseudo-spectral method combined with the fourth order Runge-Kutta time integrator with small enough time step $\delta t=2.5 \times 10^{-4}$ to conserve the energy with small enough relative error. Initial conditions are randomly drawn from the uniform distribution on a constant energy surface $E=0.1$. We parameterize the MSM model with solutions of the TBH model in (3.4) for total time $10^{5}$ as the training dataset. For the AR(p) model, 
we estimate the parameters with the same data set with discrete time step $\Delta t=0.1$ (we found worse solutions with smaller $\Delta t$ ).
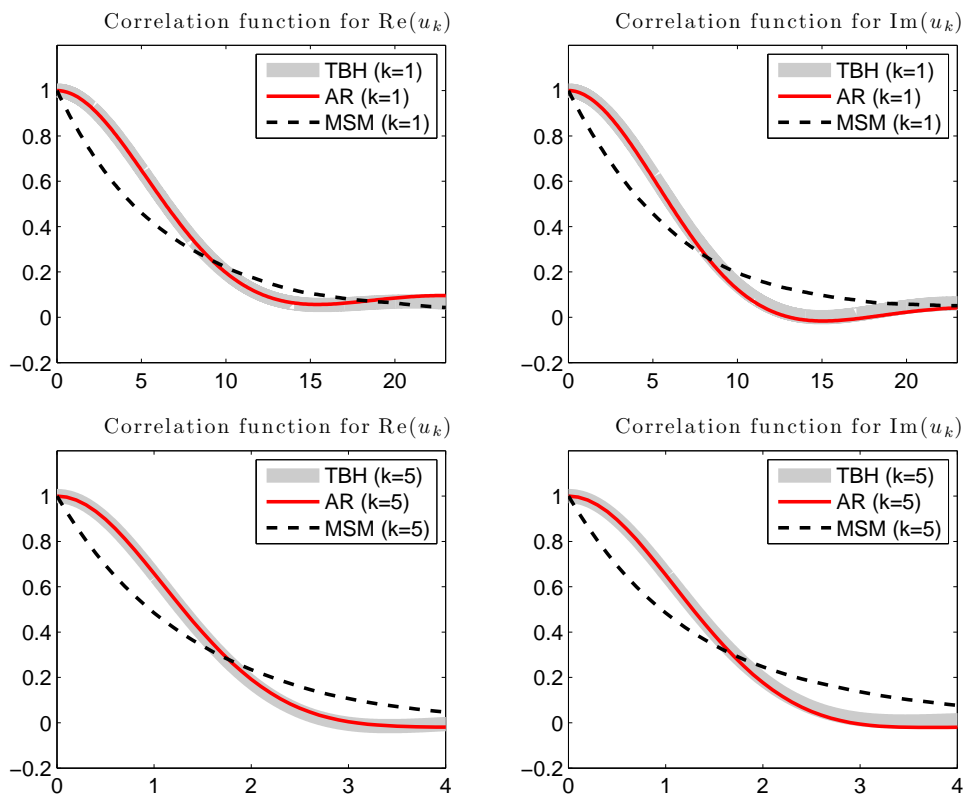

FIG. 3.5. The autocorrelation functions from the TBH model (thick grey solid curves), reproduced from the $A R(p)$ model (red solid curves), and MSM model (black dashed curves).

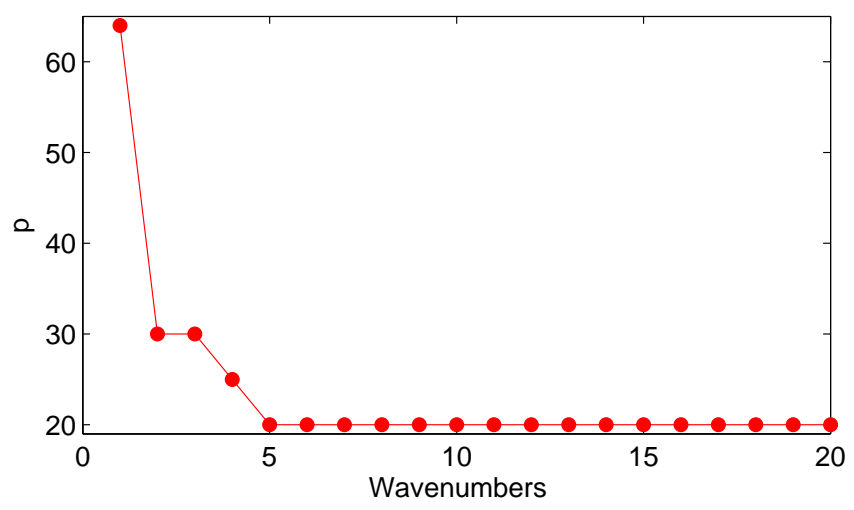

FIG. 3.6. The values of $p$ chosen in the $A R(p)$ model for the TBH model.

For the TBH case, both MSM and AR(p) models reproduce the energy spectrum $C_{k}$ perfectly (the results are not shown). In figure 3.5, we show the autocorrelation functions of wavenumbers 1 and 5 . Notice that the autocorrelation functions evolve slowly for wavenumber 1 and faster for wavenumber 5 . Meanwhile, although there is a wide range of timescales of mixing in the TBH model, all the autocorrelation functions have similar patterns with little oscillation, which has been discussed as self-similarity of correlations in [35]. Here, the $\mathrm{AR}(\mathrm{p})$ model again reproduces the autocorrelation functions much more accurately compared to the MSM model (figure 3.5). In this 
case, both the MSM and AR(p) models reproduce $T_{\text {corr }}$ and $T_{a b s}$ accurately (results are not shown); here, both correlation times, $T_{\text {corr }}$ and $T_{a b s}$, are almost identical since the autocorrelation function has little oscillation with negligible negative portion.

In figure 3.6, we show the autoregressive model order $p$ obtained through autoregressive fitting. The value of $p$ suggests that the memory depth for the lowest wave number is nearly two times longer than that of the next wavenumber. This memory assessment indeed matches the well-known fact that the correlation times of the first two modes differ by a scale factor of two [35, 36, 28].

In table 3.2 , we show the $\ell^{2}$-error and pattern correlation of the linear response estimate for the TBH case. For short time periods $(t=0.5,1.5$, and 3$)$, the associated $\ell^{2}$-errors for the response operator reproduced from the $\mathrm{AR}(\mathrm{p})$ model are much smaller than those from the MSM model. The pattern correlations for both $\mathcal{R}_{A R}(t)$ and $\mathcal{R}_{L R}(t)$ are close to 1 , indicating that the response operators produced from both the AR(p) and MSM models are nearly collinear with $\mathcal{R}(t)$, irrespective of differences in amplitudes. The infinite time linear response operator estimate with the $\operatorname{AR}(p)$ model has slightly larger $\ell^{2}$-error compared to that of the MSM model. Again, the skillful estimate for infinite time response operator with the MSM model is attributed to the correlation time fitting in (2.8), whereas the skillful estimate for finite time response with the $\mathrm{AR}(\mathrm{p})$ model is attributed to accurate fitting of the autocorrelation functions at finite time.

TABLE 3.2. $\ell^{2}$-errors and pattern correlations between the response from the TBH model and those from the $A R(p)$ and MSM models at different times.

\begin{tabular}{lcccc}
\hline \hline \multirow{2}{*}{ Time } & \multicolumn{2}{c}{$\ell^{2}$-errors } & \multicolumn{2}{c}{ Correlations } \\
& $\mathrm{AR}(\mathrm{p})$ & $\mathrm{MSM}$ & $\mathrm{AR}(\mathrm{p})$ & $\mathrm{MSM}$ \\
\hline 0.5 & 0.0035 & 0.1881 & 1.0000 & 0.9974 \\
1.5 & 0.0092 & 0.1638 & 1.0000 & 0.9978 \\
3 & 0.0136 & 0.1579 & 0.9999 & 0.9970 \\
$\infty$ & 0.0688 & 0.0327 & 0.9985 & 0.9998 \\
\hline \hline
\end{tabular}

We also consider the inviscid-unforced Lorenz-96 model (IL96) [28], the governing equation of which is given by

$$
\frac{d u_{j}}{d t}=\left(u_{j+1}-u_{j-2}\right) u_{j-1}, \quad j=1, \ldots, N
$$

with periodic boundary $u_{j+N}=u_{j}$ and $N=40$. Following [28], we numerically integrate the IL96 model with the fourth order Runge-Kutta time integrator with small enough timestep $\delta t=2^{-10}$ to ensure that the energy is conserved. Initial conditions are randomly drawn from the uniform distribution on a constant energy surface $E=1$. As in the KZ and TBH models, we parameterize the MSM model with solutions of the IL96 model in (3.5) for total time $10^{5}$ as the training dataset. For the $\mathrm{AR}(\mathrm{p})$ model, we estimate the parameters with the same data set with discrete time step $\Delta t=8 / 64$.

In this setup, the autoregressive model fitting chooses $p$ between 20-22 for each wavenumber (based on FPE criterion [5]); this indicates that the IL96 model has almost equal memory depth on every wavenumber. This conjecture is in accordance to the fact that the IL96 model has identical non-oscillatory autocorrelation functions for all wavenumbers [28]. We found that both the MSM and AR(p) models reproduce the energy spectra $C_{k}$, correlation times $T_{\text {corr }}(t)$, and $T_{a b s}(t)$ well, but the $\operatorname{AR}(\mathrm{p})$ model performs better in reproducing the autocorrelation functions than the MSM 
model (results are not shown). Similar to what we found for the TBH model, for short time periods $(t=1,2$, and 5$)$, the associated $\ell^{2}$-errors for $\mathcal{R}_{A R}(t)$ are much smaller than those for $\mathcal{R}_{L R}(t)$ (see table 3.3). The pattern correlations for both $\mathcal{R}_{A R}(t)$ and $\mathcal{R}_{M}(t)$ are close to 1 , indicating that they are both nearly collinear with $\mathcal{R}(t)$, irrespective of differences in amplitudes. Notice that at $t=\infty$, the $\ell^{2}$-error of the response estimate from the $\mathrm{AR}(\mathrm{p})$ model is comparable to that from the MSM, which confirms again the high skill of the MSM model in describing the infinite time integral of the autocorrelation function, by design.

TABLE 3.3. $\ell^{2}$-errors and correlations between the response from the IL96 model and those from from $A R(p)$ and $M S M$ at different times.

\begin{tabular}{lcccc}
\hline \multirow{2}{*}{ Time } & \multicolumn{2}{c}{$\ell^{2}$-errors } & \multicolumn{2}{c}{ Correlations } \\
& AR(p) & MSM1 & AR(p) & MSM1 \\
\hline 1 & 0.0021 & 0.1647 & 1.0000 & 0.9998 \\
2 & 0.0036 & 0.0715 & 1.0000 & 0.9996 \\
5 & 0.0165 & 0.0463 & 0.9999 & 0.9990 \\
$\infty$ & 0.0837 & 0.0953 & 0.9974 & 0.9975 \\
\hline \hline
\end{tabular}

\section{4. $\operatorname{AR}(p)$ memory models and the linear response of the forced dissi- pative L-96 model}

In Section 3, we estimate the FDT-based linear operator on three energy conserving nonlinear systems with Gaussian invariant measure. In this section, we apply the same FDT-based linear response approximation on a forced-dissipative nonlinear dynamical system, the 40-mode Lorenz 96 (L-96) model, with almost Gaussian invariant measure as $F$ increases (see figure 4.1). The non-Gaussianity of the L-96 has been recorded and analyzed in detailed in [21,33]. The governing equation of the L-96 model is as follows:

$$
\frac{d u_{j}}{d t}=\left(u_{j+1}-u_{j-2}\right) u_{j-1}-u_{j}+F, \quad j=1, \ldots, N,
$$

where the right hand side consists of an energy conserving quadratic nonlinear advective-like term, a linear dissipation, and a forcing parameter $F$, respectively. Following $[1,2,28]$, we resolve the L96 model at $N=40$ equally spaced grid points with periodic boundary to mimic weather wave patterns on a midlatitude belt. Below, we will test the skill of the $\mathrm{AR}(\mathrm{p})$ strategy for computing the response operators on various dynamical regimes ranging from weakly chaotic with $F=5,6$, to strongly chaotic with $F=8$, to the fully turbulent regime with $F=16$ (see [1,33] for the detailed chaotic measures including the Lyapunov exponent and Kolmogorov-Sinai entropy).

In our numerical experiment, we parameterize the $\mathrm{AR}(\mathrm{p})$ model with solutions at every discrete time step $\Delta t=4 / 64$ (see [22] for larger $\Delta t$ ). Figure 4.2 displays the values of $p$ chosen for the $\mathrm{AR}(\mathrm{p})$ model for various forcing strengths, $F=5,6,8$, and 16. Notice that $p$ decreases as the system becomes more chaotic or turbulent (or as $F$ increases); this is not so surprising because the correlation time decreases as $F$ increases (see $[28,1,33]$ ). Moreover, the distribution of $p$ as a function of wavenumber is highly correlated to the standard memory depth measure, absolute correlation time $T_{a b s}$, which are large on wavenumbers 8-15 for $F=5,6$ and almost flat when $F=16$ (see [28]). 

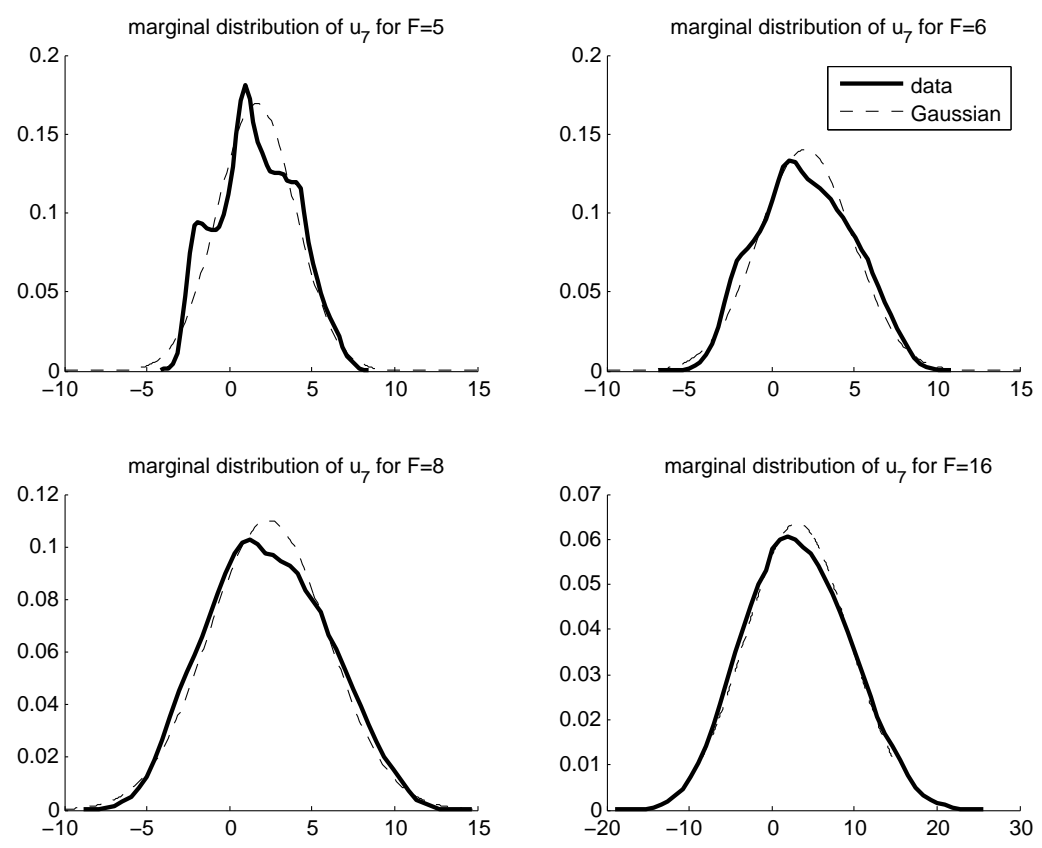

FIG. 4.1. Marginal distributions (solid) of the L-96 model for various $F$ at grid point $u_{7}$ compared to Gaussian distribution (dashes) with exactly the same mean and variance.

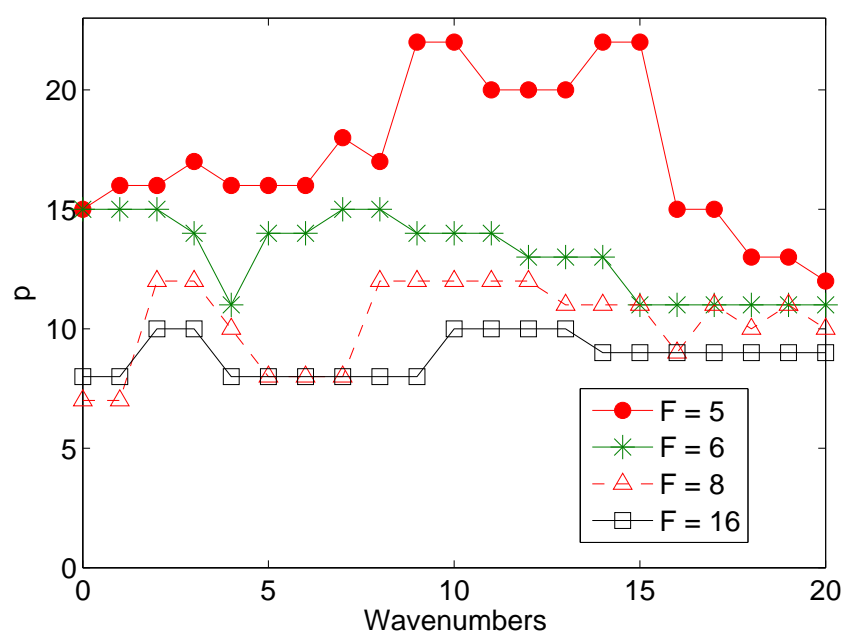

FIG. 4.2. The values of $p$ chosen in the AR(p) model for the L-96 model.

Since the invariant measure of the L-96 is not Gaussian (see figure 4.1), the linear response operator computed with (2.6) with the true time series only produces a quasi-Gaussian approximate FDT linear response. For objective diagnostic, we need to compare our approximate linear response operator with an ideal response operator that reflects the actual change of $\delta\langle A\rangle$ in (2.3). Different than the FDTbased algorithm which relies on the statistics of the unperturbed system in $(2.1)$, 

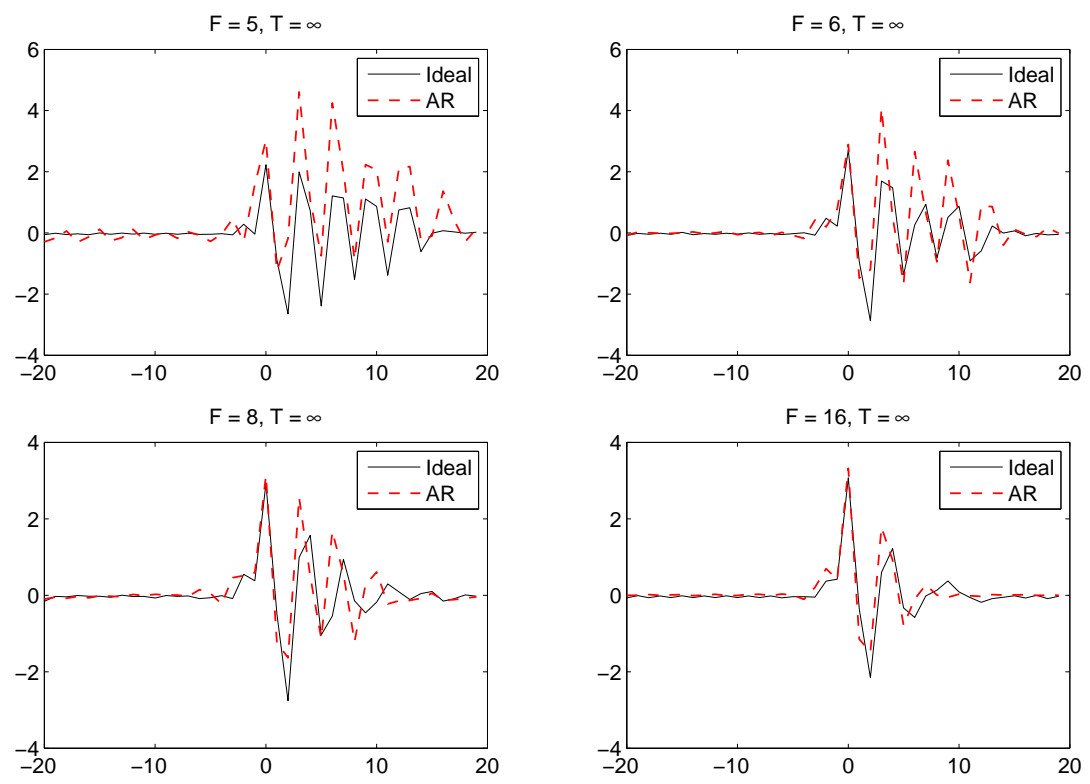

FIG. 4.3. Translationally averaged autoregressive infinite-time linear response (dashed line) and ideal response (solid line) for the L-96 model in various chaotic regimes with forcing strengths $F=5$, 6,8 , and 16 .

the computation of the ideal response involves integration of the perturbed system in (2.2), which is non-trivial and expensive [16]. In Sections 3.1 and 3.2, we ignored reporting the ideal responses for the three energy conserving systems, KZ, TBH, and IL96, since they are nearly identical to the FDT responses, $\mathcal{R}(t)$, obtained with (2.6) (see [28]).

In figure 4.3 , we show the translationally averaged infinite time linear response operators from the $\operatorname{AR}(\mathrm{p})$ model, $\mathcal{R}_{A R}(\infty)$, for various forcing strengths. It can be seen that as $F$ increases, the approximate response operator from the AR(p) model becomes closer to the ideal response operator. In the weakly chaotic regime, $F=5$ and 6 , the $\mathrm{AR}(\mathrm{p})$ response recovers the oscillations but with wrong amplitude that overshoots the ideal response. Such errors are also observed with the computationally more expensive qG-FDT response operator (see figure 2.48 in [28]).

In table 4.1, we summarize the results with pattern correlations between the ideal response and various FDT responses, including the AR(p), MSM, quasi-Gaussian response operators discussed in Section 2 and the blended response algorithm [1]. The pattern correlation of the blended response is from a version that combines a very accurate short time FDT with the qG-FDT response [1]. Note that this blended response operator is also computationally more expensive than the $\mathrm{AR}(\mathrm{p})$ response because it involves evaluating a linear tangent map at short time beyond integrating the nonlinear dynamics in (2.1) for quasi-Gaussian approximation. Generally speaking, the blended response has the highest skill among all the methods. The skill of the response with the MSM model is the lowest among all methods. In the weakly chaotic regime $(F=5,6)$, the MSM response has the lowest skill; this is attributed to severe model errors with Ornstein-Uhlenbeck approximation in the weakly chaotic regime [19]. In this regime, the skill of the $\mathrm{AR}(\mathrm{p})$ response is comparable to that of the more expensive quasi-Gaussian approximation, but they are still much less skill- 
ful compared to the blended response especially for long and infinite time. When the system is strongly chaotic $(F=8)$, the MSM, $\operatorname{AR}(\mathrm{p})$, and quasi-Gaussian strategies have comparable skills at all times. In the fully turbulent regime $(F=16)$, the model error due to the Ornstein-Uhlenbeck approximation becomes less severe and the MSM FDT response has much higher skill than before. In this regime, the $\operatorname{AR}(\mathrm{p})$ response has correlation skill beyond the quasi-Gaussian approximation and is comparable to that of the blended response at all time. The $\ell^{2}$-error of the $\mathrm{AR}(\mathrm{p})$ response, however, is only comparable to that of the quasi-Gaussian approximation (the results are not shown).

TABLE 4.1. Correlation between the ideal response and the responses from MSM, quasi-Gaussian approximation, the $A R(p)$ model, and the blended response algorithm for different rescaled times $T$ and different forcing $F$.

\begin{tabular}{rrcccc}
\hline \hline$F$ & $T$ & MSM & quasi-Gaussian & AR(p) & Blended \\
\hline 5 & 5 & 0.8268 & 0.9148 & 0.9364 & 0.9998 \\
& 20 & 0.7154 & 0.7392 & 0.7239 & 0.9996 \\
& $\infty$ & 0.6641 & 0.7322 & 0.7192 & 0.9346 \\
& & & & & \\
6 & 5 & 0.8733 & 0.9718 & 0.9564 & 0.9999 \\
& 20 & 0.6938 & 0.7866 & 0.7291 & 0.99994 \\
& $\infty$ & 0.7150 & 0.7765 & 0.7407 & 0.9827 \\
& & & & & \\
8 & 5 & 0.9429 & 0.9707 & 0.9751 & 0.9999 \\
& 20 & 0.7757 & 0.8076 & 0.7801 & 0.9976 \\
& $\infty$ & 0.7637 & 0.7618 & 0.7363 & 0.9741 \\
& & & & & \\
16 & 5 & 0.9649 & 0.9924 & 0.999 & 0.9999 \\
& 20 & 0.9136 & 0.9279 & 0.9982 & 0.9982 \\
& $\infty$ & 0.8862 & 0.9082 & 0.9892 & 0.9892 \\
\hline
\end{tabular}

\section{Concluding discussion}

In this paper, we apply linear regression models with memory, $\mathrm{AR}(\mathrm{p})$ models, to approximate linear response for the mean to a constant change in external forcing. From numerical tests on three energy conserving nonlinear chaotic dynamical systems with Gaussian invariant measure (Section 3), we conclude that the response estimate with the $\mathrm{AR}(\mathrm{p})$ model is much more skillful than that with the MSM for short time. The improved skill in short time response estimate with the AR(p) model over the MSM is attributed to more accurate fitting of the autocorrelation functions. For infinite time response, the improved estimate with the $\mathrm{AR}(\mathrm{p})$ model over the MSM becomes negligible since the parameters in the MSM are directly fitted to the correlation time (see (2.8)). We also see high pattern correlation between distributions of autoregressive order $p$ and memory depth measure $T_{a b s}$ as functions of wavenumbers, which suggests that the $\mathrm{AR}(\mathrm{p})$ regression fitting $[7,5]$ automatically informs us about the memory depth of the system through the choice of the autoregressive order $p$ without having to compute $T_{a b s}$.

We also test this $\mathrm{AR}(\mathrm{p})$ strategy on a dissipative-forced nonlinear system, the L-96 model with an invariant measure which is slightly skewed from the Gaussian distribution, on various dynamical regimes ranging from weakly to strongly chaotic to fully turbulent (Section 4). In this context, the $\mathrm{AR}(\mathrm{p})$ model produces a response 
estimate that is much more skillful than that of the MSM particularly in the weakly chaotic regime. The skill separation between the AR(p) and MSM decreases as the system becomes more chaotic; this phenomenon is also observed in the context of climate modeling $[38,37,39,11]$ and filtering $[22,19,20,23]$. We also find that the skill of the response from the $\mathrm{AR}(\mathrm{p})$ model is comparable to that of the more expensive quasi-Gaussian approximation in all three dynamical regimes: the weakly choatic, strongly chaotic, and fully turbulent regimes.

Finally, we should point out that the impressive skill with the computationally cheap $\operatorname{AR}(p)$ model is only attainable when a longer time series is available. Such a restriction appears because the AR(p) models have more coefficients (larger $p$ ) especially when the signals being modeled have long memory.

Acknowledgment. The authors thank R. Abramov for sharing the ideal response dataset for the L-96 model in Section 4. The research of E.L.K. is partially supported by the Faculty Research Grant Program from the University Research Council at University of Cincinnati and the Charles Phelps Taft Research Center. The research of J.H. is partially supported by the Office of Naval Research Grant N00014-11-1-0310, the NC State startup fund, and the NC State Faculty Research and Professional Development fund. The research of A.J.M. is partially supported by the National Science Foundation Grant DMS-0456713 and the Office of Naval Research Grants ONR DRI N00014-10-1-0554 and N00014-11-1-0306.

\section{REFERENCES}

[1] R.V. Abramov and A.J. Majda, Blended response algorithm for linear fluctuation-dissipation for complex nonlinear dynamical systems, Nonlin., 20, 2793-2821, 2007.

[2] R.V. Abramov and A.J. Majda, New approximations and tests of linear fluctuation-response for chaotic nonlinear forced-dissipative dynamical systems, J. Non. Sci., 18, 303-341, 2008.

[3] R.V. Abramov and A.J. Majda, A new algorithm for low-frequency climate response, J. Atmos. Sci., 66, 286-309, 2009.

[4] R.V. Abramov and A.J. Majda, Low-frequency climate response of quasigeostrophic wind-driven ocean circulation, J. Phys. Ocean., 42, 243-260, 2011.

[5] H. Akaike, Fitting autoregressive models for regression, Ann. Inst. Stat. Math., 21, 243-247, 1969.

[6] R. Balescu, Statistical Dynamics: Matter Out of Equilibrium, Imperial College Press, London, 1997.

[7] P.J. Brockwell and R.A. Davis, Introduction to Time Series and Forecasting, Springer Verlag, 2002.

[8] U. Deker and F. Haake, Fluctuation-dissipation theorems for classical processes, Phys. Rev. A, 11, 2043-2056, 1975.

[9] T. DelSole, A fundamental limitation of Markov models, J. Atmos. Sci., 57, 2158-2168, 2000.

[10] T. DelSole, Stochastic model of quasigeostrophic turbulence, Surv. Geophys., 25, 107-149, 2004.

[11] C. Franzke and A.J. Majda, Low-order stochastic mode reduction for a prototype atmospheric GCM, J. Atmos. Sci., 63, 457-479, 2006.

[12] C.W. Gardiner, Handbook of Stochastic Methods for Physics, Chemistry, and the Natural Sciences, Springer-Verlag, New York, 3rd edition, 2004.

[13] B. Gershgorin and A.J. Majda, Filtering a nonlinear slow-fast system with strong fast forcing, Commun. Math. Sci., 8, 67-92, 2010.

[14] A. Gritsun and G. Branstator, Climate response using a three-dimensional operator based on the fluctuation-dissipation theorem, J. Atmos. Sci., 64, 2558-2575, 2007.

[15] A. Gritsun, G. Branstator, and A.J. Majda, Climate response of linear and quadratic functionals using the fluctuation-dissipation theorem, J. Atmos. Sci., 65, 2824-2841, 2008.

[16] A. Gritsun and V. Dymnikov, Barotropic atmosphere response to small external actions: Theory and numerical experiments, Izv. Acad. Sci. USSR, Atmos. Oceanic Phys., 35, 511-525, 1999.

[17] A. Gritsun and V. Dymnikov, Construction of the linear response operator of an atmospheric general circulation model to small external forcing, Russ. J Numer. Anal. Math. Modell., 17, 399-416, 2002. 
[18] M. Hairer and A.J. Majda, A simple framework to justify linear response theory, Nonlin., 23, 909, 2010.

[19] J. Harlim and A.J. Majda, Filtering nonlinear dynamical systems with linear stochastic models, Nonlin., 21, 1281-1306, 2008.

[20] J. Harlim and A.J. Majda, Catastrophic filter divergence in filtering nonlinear dissipative systems, Commun. Math. Sci., 8, 27-43, 2010.

[21] K. Haven, A.J. Majda, and R. Abramov, Quantifying predictability through information theory: Small sample estimation in a non-gaussian framework, J. Comp. Phys., 206, 334-362, 2005.

[22] E.L. Kang and J. Harlim, Filtering nonlinear spatio-temporal chaos with autoregressive linear stochastic models, Physica D, 241(12), 1099-1113, 2012.

[23] S.R. Keating, A.J. Majda, and K.S. Smith, New methods for estimating ocean eddy heat transport using satellite altimetry, Monthly Weather Review, 140(5), 1703-1722, 2012.

[24] R. Kubo, M. Toda, and N. Hashitsume, Statistical Physics II: Nonequilibrium Statistical Mechanics, Springer Series in Solid-State Sciences, Springer, 1985.

[25] C.E. Leith, Climate response and fluctuation dissipation, J. Atmos. Sci., 32, 2022-2026, 1975.

[26] E.N. Lorenz, Predictability - a problem partly solved, in Proceedings on Predictability, held at ECMWF on 4-8 September, 1995, 1-18, 1996.

[27] A.J. Majda, R. Abramov, and B. Gershgorin, High skill in low frequency climate response through fluctuation-dissipation theorems despite structural instability, Proc. Nat. Acad. Sci., 107, 581-586, 2010.

[28] A.J. Majda, R.V. Abramov, and M.J. Grote, Information Theory and Stochastics for Multiscale Nonlinear Systems, CRM Monograph Series v.25, American Mathematical Society, Providence, Rhode Island, USA, 2005.

[29] A.J. Majda and B. Gershgorin, Quantifying uncertainty in climate change science through empirical information theory, Proc. Nat. Acad. Sci. USA, 107, 14958-14963, 2010.

[30] A.J. Majda and B. Gershgorin, Improving model fidelity and sensitivity for complex systems through empirical information theory, Proc. Nat. Acad. Sci. USA, 108, 10044-10049, 2011.

[31] A.J. Majda and B. Gershgorin, Link between statistical equilibrium fidelity and forecasting skill for complex systems with model error, Proc. Nat. Acad. Sci. USA, 108, 12599-12604, 2011.

[32] A.J. Majda, B. Gershgorin, and Y. Yuan, Low frequency response and fluctuation-dissipation theorems: Theory and practice, J. Atmos. Sci., 67, 1181-1201,2010.

[33] A.J. Majda and J. Harlim, Filtering Complex Turbulent Systems, Cambridge University Press, $\mathrm{UK}, 2012$.

[34] A.J. Majda, J. Harlim, and B. Gershgorin, Mathematical strategies for filtering turbulent dynamical systems, Disc. Cont. Dyn. Sys. A, 27, 441-486, 2010.

[35] A.J. Majda and I. Timofeyev, Remarkable statistical behavior for truncated Burgers-Hopf dynamics, Proc. Nat. Acad. Sci., 97, 12413-12417, 2000.

[36] A.J. Majda and I. Timofeyev, Statistical mechanics for truncations of the Burgers-Hopf equation: a model for intrinsic behavior with scaling, Milan J. Math., 70, 39-96, 2002.

[37] A.J. Majda and I. Timofeyev, Low dimensional chaotic dynamics versus intrinsic stochastic chaos: A paradigm model, Physica D, 199, 339-368, 2004.

[38] A.J. Majda, I. Timofeyev, and E. Vanden-Eijnden, A mathematical framework for stochastic climate models, Commun. Pure Appl. Math., 54, 891-974, 2001.

[39] A.J. Majda, I. Timofeyev, and E. Vanden-Eijnden, Stochastic models for selected slow variables in large deterministic systems, Nonlin., 19, 769-794, 2006.

[40] A.J. Majda and X. Wang, Linear response theory for statistical ensembles in complex systems with time-periodic forcing, Commun. Math. Sci., 8, 187-216, 2010.

[41] C. Penland, Random forcing and forecasting using principal oscillation pattern analysis, Monthly Weather Review, 117, 2165-2185, 1989.

[42] C. Penland and P.D. Sardeshmukh, The optimal growth of tropical sea surface temperature anomalies, J. Climate, 8, 1999-2024, 1995.

[43] H. Risken, The Fokker-Planck Equation: Methods of Solution and Applications, Springer Series in Synergetics, Springer, 1989.

[44] N.J. Zabusky and M.D. Kruskal, Interaction of "solitons" in a collisionless plasma and the recurrence of initial states, Phys. Rev. Lett., 15, 240-243, 1965. 